Original article

\title{
Direct medical costs of ADHD and its comorbid conditions on basis of a claims data analysis
}

\author{
Berit Libutzki ${ }^{\mathrm{a}, *}$, Saskia Ludwig ${ }^{\mathrm{a}}$, Melanie May ${ }^{\mathrm{a}}$, Rasmus Højbjerg Jacobsen ${ }^{\mathrm{b}}$, \\ Andreas Reif ${ }^{\mathcal{C}}$, Catharina A. Hartman ${ }^{\mathrm{d}}$ \\ ${ }^{a}$ HGC Healthcare Consultants GmbH, Graf-Adolf-Platz 15, D-40213, Düsseldorf, Germany \\ ${ }^{\mathrm{b}}$ VIVE - The Danish Center for Social Science Research. Herluf Trolles Gade 11, DK-1052, København K, Denmark \\ ${ }^{\mathrm{c}}$ Department for Psychiatry, Psychosomatic Medicine and Psychotherapy, University Hospital Frankfurt, Heinrich-Hoffmann-Str. 10, 60528, Frankfurt am Main, \\ Germany \\ d Department for Psychiatry, University of Groningen, University Medical Center Groningen, Hanzeplein 1 code CC72, 9713 GZ, Groningen, the Netherlands
}

\section{A R T I C L E I N F O}

\section{Article history:}

Received 23 October 2018

Received in revised form 31 January 2019

Accepted 31 January 2019

Available online 22 February 2019

\section{Keywords:}

Attention deficit hyperactivity disorder (ADHD)

Real-world evidence (RWE)

Direct costs

Comorbidities

Psychiatric disorders

Obesity

\begin{abstract}
A B S T R A C T
Background: ADHD is a highly prevalent disease in childhood which often persists into adulthood, then co-occurring with common adult conditions. Especially for adult ADHD, little is known about the costs of ADHD and the additional costs of comorbid conditions.

Aims: To determine medical costs of ADHD and costs of comorbidities (mood, anxiety and substance use disorders, obesity), including their co-occurrence rates, stratified by age and gender.

Method: Claims data from a German Statutory Health Insurance database with approximately four million member-records per year were analysed. A total of 25,300 prevalent ADHD patients were identified by means of an ICD-10 GM diagnosis of ADHD. A 1:1 age and gender adjusted reference group without ADHD diagnosis was randomly selected. Total health claims and health care costs related to ADHD were analysed, in addition to more targeted analyses of the occurrence and costs of pre-defined common comorbidities of, in particular, adult ADHD (SUD, mood and anxiety disorders, obesity). Outcomes were mean costs per patient and occurrence rates of comorbid conditions. Surplus costs of a comorbid condition in persons with ADHD relative to costs of this condition in persons without ADHD were calculated. Subgroup analyses were conducted based on age (0-12 years, 13-17 years, 18-30years, $30+$ years) and gender.

Results: Patients with ADHD were $€ 1500$ more expensive annually than individuals without ADHD $(\mathrm{p}<0.001)$. Main cost drivers were inpatient care, psychiatrists and psychotherapists. Mood, anxiety, substance use disorders and obesity were significantly more frequent in ADHD patients and additional costs resulting from the comorbid conditions amounted up to $€ 2800$. Costs were slightly higher in women than men and increased with age for both genders. In young adults (18-30 years) health care costs dropped notably, especially costs for the medical treatment of ADHD with stimulants and costs for psychiatrists, before rising again in the group of patients over 30 years who had higher comorbidity rates. Conclusions: Medical costs for ADHD are substantial, in part through frequently occurring comorbid conditions, and particularly in adulthood, and are likely to further accelerate in the coming years. A gap of care was found, starting with the transition age group of patients over 17 years, as indicated by reduced costs per person during young adulthood, as well as an overall strong drop in administrative prevalence. In the future, approaches to improve the situation of care and reduce costs at the same time, i.e. through managed care programmes, should be implemented and benefit from detailed knowledge on age and gender-specific cost-drivers.
\end{abstract}

(c) 2019 Elsevier Masson SAS. All rights reserved.

\footnotetext{
* Corresponding author.

E-mail address: berit.libutzki@hgc-team.de (B. Libutzki).
}

\section{Introduction}

Over the last years, adult ADHD has been increasingly recognized as an impairing mental health condition [1]. Globally, administrative prevalence has increased substantially [6-9], resulting in rising total costs of ADHD in Germany and the United 
States (US) $[6,10]$. Worldwide, ADHD is associated with a high economic burden for children and young adults amounting up to $\$ 1000$ incremental costs per ADHD patient in the US [2] and up to $€ 2900$ surplus costs in Germany for ADHD patients regardless of age [3]. Current literature shows that the costs of ADHD in adulthood have been much less investigated than ADHD in childhood, which is an important gap in literature.

Aside from the costs incurred from ADHD directly, ADHD is associated in both childhood and adulthood with multiple comorbidities [11]. Frequent comorbidities across the lifespan but particularly in adulthood are obesity, substance use disorders (SUD), mood disorders and anxiety disorders $[12,13,3,14,15]$. It is challenging to differentiate ADHD from other psychiatric conditions, given overlapping or very similar symptoms, especially in adult ADHD patients [4]. Nonetheless, comorbidities with obesity, SUDs, mood and anxiety disorders have been well-established. Comorbid conditions of ADHD are considered main cost drivers in ADHD patients [4,5] but this needs more study.

In fact, studies presenting costs for higher use of healthcare services in relation to these co-occurring conditions in ADHD have not yet been conducted. In addition, detailed subgroup analyses, stratifying for age and gender are scarce.

To obtain detailed insight into the costs of ADHD across the lifespan, and separately for men and women, very large samples representative of the general population, which include a large number of ADHD patients, are needed, such as claims data or register data. Detailed cost estimations are of high relevance for health insurances, and for national economies as a whole to identify gaps in care and develop preventive measures or care programmes which address these gaps. There is thus a need for identifying ADHD's main cost drivers, stratified by age and gender. This goes hand in hand with the need to document the burden of these comorbidities which trigger both a decrease in the quality of life of ADHD patients and high surplus costs for payers.

The aim of the present study therefore is to assess first, the overall direct medical costs of ADHD stratified by age and gender and, second, to zoom further in and depict the surplus costs of the four pre-defined comorbidities, stratified by age and gender.

\section{Method}

\subsection{Study design and participants}

The study is conducted based on claims data from a German Statutory Health Insurance (SHI) database. Approximately $90 \%$ of the German population is insured in SHIs, thus SHI databases are an important source of data. The SHI database used contains approximately four million member-records per year from over 60 nationwide German SHIs (from a total of 118 SHIs). Data on health claims are transferred directly from healthcare providers to specialized data centres owned by SHIs, where all data are anonymized before entering the database. The sample is age-and gender-adjusted to the German population. There is a good overall accordance of the database and the German population in terms of morbidity, mortality and drug usage [16]. Routine billing data over a period of six years (2009-2014) are analysed. For 2014 the study population comprizes $3,705,952$ individuals. The inclusion criteria are set in such a way that in each year considered, i.e. 2009-2014, the individual receives at least one inpatient or day patient main diagnosis of ADHD or two outpatient ADHD diagnoses (according to ICD-10 coding F90.0, F90.1 and F90.8) in three different quarters of the year to select patients with a secure diagnosis. To compare this group of individuals with ADHD to individuals without ADHD, a 1:1 age and gender adjusted reference group with individuals without an ADHD diagnosis between 2009-2014 is randomly selected from the total database. Two exclusion criteria are applied: first, individuals have to be continuously insured throughout the observation period to avoid a bias through loss to follow-up. Second, individuals with high-cost diagnoses are excluded when incurring at least five times the established threshold of $€ 3651.16$ by the 'Risikostrukturausgleich' (SHI morbidity-adjusted risk adjustment scheme) to avoid a bias in costs due to outliers. Applying these inclusion and exclusion criteria, a total of 25,300 individuals with an ADHD diagnosis in 2014 and 25,300 individuals without an ADHD diagnosis are identified for further analyses.

\subsection{Outcomes and statistical analyses}

To describe the study population, basic demographic information is extracted for all identified ADHD patients and individuals of the reference group in 2014 and further analyses are conducted in gender and age stratified subgroups ( 0 -12 years, $13-17$ years, 1830 years and $\geq 31$ years). Prevalence rates are calculated based on the total number of insured persons in the database and the number of diagnosed ADHD patients in the subgroup. One aim of the study is to assess direct medical costs of ADHD. Here we calculate mean direct costs in ADHD patients and individuals in the reference group without ADHD for (1) inpatient and day-patient care, (2) outpatient care: general practitioners (GP), internists, paediatricians, psychiatrists and accredited psychotherapists, (3) remedies: therapeutic devices, aids and occupational therapy/ behavioural therapy, (4) medical prescriptions: stimulants (coded via the German anatomical-therapeutic-chemical (ATC) N06BA) and other drugs prescribed by a medical doctor, and (5) sick payment for $\geq 42$ working days (shorter sick leaves cannot be extracted from the German claims data because up to 42 days the employer continues to pay normal wages). All costs in the beforedefined categories triggered by any comorbidity are included in the cost analysis. The second aim is to analyse the burden of ADHD with comorbidities, stratified by age and gender. So in addition to studying the costs of ADHD as such, all identified ADHD patients and individuals in the reference group without ADHD are additionally scanned for the following comorbidities, which are known to be associated with adult ADHD: substance use disorder (SUD) (ICD-10-GM: F10-F19); morbid obesity (ICD-10-GM: E66); mood disorders (ICD-10-GM: F30-F39); and anxiety disorders (ICD-10-GM: F40, F41, F43, F45). The occurrence of these comorbidities is measured in ADHD patients and individuals in the reference group without ADHD; Odds Ratios (OR) are used to quantify the odds to manifest comorbidities for ADHD and reference patients. Further, the surplus health care costs of these four pre-defined conditions associated with ADHD are of interest. Surplus costs of a frequent co-occurring condition are assessed by calculating the difference between mean health care costs of individuals with ADHD and a co-occurring condition, and mean health care costs of individuals in the reference group without ADHD but with the same condition. Differences in costs and resource utilization between groups are tested using the MannWhitney $U$ Test. Statistical significance is determined at the level of $\mathrm{p} \leq 0.05$. ORs are used to quantify the odds to manifest comorbidities for ADHD and reference patients. All patient-level data in the database are de-identified to comply with German data protection regulations. Use of the study database for health services research is therefore fully compliant with German federal law and, accordingly, no institutional review board/ethical approval and informed consent of the patient was needed. For data storage and processing, Microsoft Office Excel ${ }^{\circledR} 2010$ (Microsoft Corporation, WA, USA) and SAS ${ }^{\circledR}$ (Version 9.2; SAS Institute Inc., NC, USA) are used. 
Table 1

Prevalence of ADHD stratified by age groups and gender in the year 2014 .

\begin{tabular}{|c|c|c|c|c|c|c|c|c|c|}
\hline \multirow{2}{*}{$\begin{array}{l}\text { Age groups } \\
\text { (years) }\end{array}$} & \multicolumn{3}{|l|}{ female } & \multicolumn{3}{|l|}{ male } & \multicolumn{3}{|c|}{ Both gender } \\
\hline & No ADHD & ADHD & prevalence & No ADHD & ADHD & prevalence & No ADHD & ADHD & prevalence \\
\hline 0-12 y. & 202,270 & 2,924 & $1.45 \%$ & 212,670 & 9,058 & $4.26 \%$ & 414,940 & 11,982 & $2.89 \%$ \\
\hline $13-17 \mathrm{y}$ & 92,320 & 1,806 & $1.96 \%$ & 97,846 & 6,042 & $6.18 \%$ & 190,166 & 7,848 & $4.13 \%$ \\
\hline $18-30 \mathrm{y}$. & 260,209 & 940 & $0.36 \%$ & 265,498 & 2,549 & $0.96 \%$ & 525.707 & 3.489 & $0.66 \%$ \\
\hline$\geq 31 \mathrm{y}$ & $1,343,924$ & 916 & $0.07 \%$ & $1,231,215$ & 1,065 & $0.09 \%$ & $2,575,139$ & 1,981 & $0.08 \%$ \\
\hline Total & $1,898,723$ & 6,586 & $0.35 \%$ & $1,807,229$ & 18,714 & $1.04 \%$ & $3,705,952$ & 25,300 & $0.68 \%$ \\
\hline
\end{tabular}

\section{Results}

\subsection{Clinical and demographic data}

Of 3,705,952 individuals in the database 25,300 are diagnosed with ADHD, of which 18,714 are male and 6586 are female (Table 1 ). This yields an overall prevalence of $0.68 \%$ across the full lifespan. The prevalence estimates for the age group 0-12 years (2.89\%) and age group $13-17$ years (4.13\%) are over three times higher than in the adult individuals (18-30 y.: $0.66 \%$ and $\geq 31 \mathrm{y}$ : $0.08 \%$ ). Female ADHD patients are on average 17.7 years old ( $\mathrm{SD} \pm 12.9$, median 13 , LQ 10 , UQ 18 , range $0-96$ ), whereas male ADHD patients are younger at a mean of 14.7 years $(S D \pm 8.8$, median 13, LQ 10, UQ 16, range 2-94). This illustrates that the distribution of the administrative ADHD prevalence in the SHI database is much higher in younger patients with ADHD than adults.

\subsection{Medical costs}

Table 2 shows that, overall, ADHD patients trigger significantly higher healthcare costs than individuals of the reference group without ADHD. Over all age groups there are surplus costs of $€ 1508$ ( $\mathrm{p}$-value < 0.001). The main cost driver is hospital costs, i.e. inpatient care, at $€ 648$ additional costs per ADHD patient per year, which accounts for approx. $43 \%$ of the overall surplus costs. Hospital costs are followed by visits to psychiatrists (accounting for $16 \%$ of the total surplus costs) at $€ 243$ surplus costs, which roughly corresponds to four times the quarterly allowance for psychiatrists in Germany (i.e., on average, an ADHD patient sees a psychiatrist at least once every quarter). On position three, stimulant costs as expenses for ADHD-specific medication follow at $€ 168$ surplus costs ( $11 \%$ of the total surplus costs). Visits at the GP and occupational therapy are cost factors as well, whereas sick payment, aid costs and remedies show surpluses of less than $€ 50$.
In the 'other drugs' category, ADHD patients trigger overall slightly lower costs than individuals without ADHD (i.e. difference of $€ 8$ ). Stratified by age groups, the cost data show that medical costs increase with age. In the ADHD group costs were highest in the age group $31+$ years at $€ 3402$, which compares to costs of $€ 1220$ in the reference group, leading to surplus costs of $€ 2182$ per ADHD patient per year. Psychiatrist costs increase with age for ADHD patients, i.e., more than twice as high for adults aged over 31 years compared to young adults between 18-30 years. The opposite can be seen in occupational therapy costs, which are triggered mostly by underage ADHD patients. Stimulant costs are highest in teenagers with ADHD (13-17 years) at $€ 215$ and decrease in the transition age group of $18-30$ years to $€ 151$ before slightly increasing again to $€ 181$ for adults over 31 years of age. The aforementioned lower costs for other drugs only hold for children and adolescents but not for adults.

\subsection{Comorbid conditions and comorbidity-triggered costs}

Patients with ADHD are significantly more likely to suffer from SUD, obesity, mood and anxiety disorders than the reference group without ADHD (Table 3). Over all age groups, 1097 ADHD patients suffer from SUD (in reference group 324), 2041 from obesity (in reference group 1140), 3231 from mood disorders (in reference group 639) and 6417 from anxiety disorders (in reference group 1962). The OR in the full sample is 3 to 6 times higher in ADHD patients (SUD: 3.5; obesity: 1.9; $\operatorname{mood}$ disorders 5.7; anxiety disorders 4.0). A distinct gender specificity can be identified: in the reference group, which is representative for the general population, SUD is more prevalent in male than female individuals without ADHD, but in the ADHD group a higher proportion of female ADHD patients have SUD than male ADHD patients, as can be seen also by the OR (females 3.7; males 3.4). This effect is most visible in the age group of 13-17 years, where females have an OR of 4.8 and males of 2.9. The OR for morbid obesity on the other

Table 2

Direct medical costs $[€]$ of patients with ADHD and individuals without ADHD ${ }^{\mathrm{a}}$ in 2014 stratified by age groups.

\begin{tabular}{|c|c|c|c|c|c|c|c|c|c|c|c|c|c|c|c|}
\hline \multirow[t]{2}{*}{ Costs $[€]$} & \multicolumn{3}{|c|}{$0-12$ years } & \multicolumn{3}{|c|}{ 13-17 years } & \multicolumn{3}{|c|}{$18-30$ years } & \multicolumn{3}{|c|}{$\geq 31$ years } & \multicolumn{3}{|c|}{ all age groups } \\
\hline & $\begin{array}{l}\text { no } \\
\text { ADHD }\end{array}$ & ADHD & $\begin{array}{l}\text { surplus } \\
\text { costs }\end{array}$ & $\begin{array}{l}\text { no } \\
\text { ADHD }\end{array}$ & ADHD & $\begin{array}{l}\text { surplus } \\
\text { costs }\end{array}$ & $\begin{array}{l}\text { no } \\
\text { ADHD }\end{array}$ & ADHD & $\begin{array}{l}\text { surplus } \\
\text { costs }\end{array}$ & $\begin{array}{l}\text { no } \\
\text { ADHD }\end{array}$ & ADHD & $\begin{array}{l}\text { surplus } \\
\text { costs }\end{array}$ & $\begin{array}{l}\text { no } \\
\text { ADHD }\end{array}$ & ADHD & $\begin{array}{l}\text { surplus } \\
\text { costs }\end{array}$ \\
\hline Hospital & 113 & 673 & 561 & 275 & 1,010 & 734 & 311 & 930 & 619 & 451 & 1,319 & 868 & 227 & 876 & 648 \\
\hline $\begin{array}{l}\text { General } \\
\text { practitioner }\end{array}$ & 127 & 242 & 116 & 103 & 213 & 110 & 104 & 198 & 94 & 159 & 280 & 121 & 120 & 231 & 111 \\
\hline Psychotherapist & 23 & 154 & 130 & 24 & 130 & 105 & 35 & 140 & 104 & 38 & 272 & 234 & 27 & 157 & 130 \\
\hline Psychiatrist & 18 & 308 & 290 & 19 & 277 & 258 & 11 & 143 & 132 & 15 & 145 & 130 & 17 & 259 & 243 \\
\hline Stimulant & 0 & 139 & 139 & 0 & 215 & 215 & 2 & 151 & 149 & 0 & 181 & 181 & 1 & 168 & 168 \\
\hline Other drugs & 216 & 141 & -75 & 230 & 209 & -21 & 162 & 230 & 68 & 309 & 621 & 313 & 228 & 220 & -8 \\
\hline Sick payment & 0 & 0 & 0 & 0 & 0 & 0 & 14 & 76 & 62 & 120 & 367 & 246 & 11 & 45 & 34 \\
\hline Aid & 31 & 54 & 23 & 49 & 40 & -9 & 33 & 25 & -8 & 51 & 77 & 27 & 39 & 48 & 9 \\
\hline $\begin{array}{l}\text { Occupational } \\
\text { therapy }\end{array}$ & 23 & 258 & 234 & 5 & 40 & 35 & 2 & 21 & 19 & 9 & 48 & 39 & 13 & 140 & 127 \\
\hline Remedies & 55 & 131 & 76 & 32 & 51 & 19 & 22 & 37 & 15 & 69 & 93 & 25 & 45 & 91 & 47 \\
\hline Total & 606 & 2,100 & 1,494 & 738 & 2,185 & 1,447 & 696 & 1,950 & 1,255 & 1,220 & 3,402 & 2,182 & 728 & 2,236 & 1,508 \\
\hline
\end{tabular}

a ADHD and reference group without ADHD 1:1 age and gender adjusted. 
Table 3

Prevalence and Odds Ratios of comorbid conditions in ADHD patients and reference group without ADHD ${ }^{\mathrm{a}}$ in 2014 stratified by gender and age groups.

\begin{tabular}{|c|c|c|c|c|c|c|c|c|c|c|c|c|c|c|c|}
\hline & \multicolumn{3}{|l|}{$0-12$ years } & \multicolumn{3}{|c|}{ 13-17 years } & \multicolumn{3}{|c|}{$18-30$ years } & \multicolumn{3}{|l|}{$\geq 31$ years } & \multicolumn{3}{|c|}{ all age groups } \\
\hline & no ADHD & ADHD & OR & no ADHD & ADHD & OR & no ADHD & ADHD & OR & no ADHD & ADHD & OR & no ADHD & ADHD & OR \\
\hline \multicolumn{16}{|l|}{ Female $^{a}$} \\
\hline SUD & $0.0 \%$ & $0.0 \%$ & & $0.8 \%$ & $3.6 \%$ & 4.8 & $3.8 \%$ & $11.2 \%$ & 3.2 & $5.1 \%$ & $19.5 \%$ & 4.5 & $1.5 \%$ & $5.3 \%$ & 3.7 \\
\hline Mood disorder & $0.3 \%$ & $3.5 \%$ & 13.0 & $2.9 \%$ & $12.3 \%$ & 4.7 & $8.3 \%$ & $36.5 \%$ & 6.3 & $18.6 \%$ & $70.3 \%$ & 10.4 & $4.7 \%$ & $19.9 \%$ & 5.0 \\
\hline Anxiety disorder & $5.4 \%$ & $20.6 \%$ & 4.6 & $11.6 \%$ & $32.2 \%$ & 3.6 & $18.9 \%$ & $49.8 \%$ & 4.2 & $28.3 \%$ & $62.9 \%$ & 4.3 & $12.2 \%$ & $33.8 \%$ & 3.7 \\
\hline Obesity & $4.3 \%$ & $7.4 \%$ & 1.8 & $4.7 \%$ & $10.2 \%$ & 2.3 & $5.5 \%$ & $11.5 \%$ & 2.2 & $12.6 \%$ & $15.8 \%$ & 1.3 & $5.7 \%$ & $9.9 \%$ & 1.8 \\
\hline \multicolumn{16}{|l|}{ Male $^{a}$} \\
\hline SUD & $0.1 \%$ & $0.3 \%$ & 4.6 & $0.8 \%$ & $2.3 \%$ & 2.9 & $3.7 \%$ & $11.9 \%$ & 3.5 & $7.4 \%$ & $26.3 \%$ & 4.5 & $3.4 \%$ & $4.0 \%$ & 3.4 \\
\hline Mood disorder & $0.6 \%$ & $3.4 \%$ & 6.0 & $1.3 \%$ & $6.1 \%$ & 5.1 & $4.0 \%$ & $21.2 \%$ & 6.5 & $9.2 \%$ & $65.6 \%$ & 18.8 & $1.8 \%$ & $10.3 \%$ & 6.4 \\
\hline Anxiety disorder & $5.3 \%$ & $18.7 \%$ & 4.1 & $5.2 \%$ & $19.0 \%$ & 4.3 & $8.8 \%$ & $29.8 \%$ & 4.4 & $13.2 \%$ & $55.5 \%$ & 8.2 & $6.2 \%$ & $22.4 \%$ & 4.4 \\
\hline Obesity & $4.2 \%$ & $6.4 \%$ & 1.6 & $4.0 \%$ & $8.5 \%$ & 2.2 & $2.6 \%$ & $7.0 \%$ & 2.8 & $7.0 \%$ & $10.8 \%$ & 1.6 & $4.1 \%$ & $7.4 \%$ & 1.9 \\
\hline \multicolumn{16}{|l|}{ Both gender } \\
\hline SUD & $0 \%$ & $0 \%$ & 4.6 & $0.8 \%$ & $2.6 \%$ & 3.3 & $3.7 \%$ & $11.7 \%$ & 3.4 & $6.4 \%$ & $23.2 \%$ & 4.4 & $1.3 \%$ & $4.3 \%$ & 3.5 \\
\hline Mood disorder & $1 \%$ & $3 \%$ & 6.9 & $1.7 \%$ & $7.6 \%$ & 4.9 & $5.2 \%$ & $25.3 \%$ & 6.2 & $13.5 \%$ & $67.8 \%$ & 13.5 & $2.5 \%$ & $12.8 \%$ & 5.7 \\
\hline Anxiety disorder & $5 \%$ & $19 \%$ & 4.2 & $6.7 \%$ & $22.1 \%$ & 3.9 & $11.6 \%$ & $35.2 \%$ & 4.2 & $20.2 \%$ & $58.9 \%$ & 5.7 & $7.8 \%$ & $25.4 \%$ & 4.0 \\
\hline Obesity & $4 \%$ & $7 \%$ & 1.6 & $4.2 \%$ & $8.9 \%$ & 2.3 & $3.4 \%$ & $8.2 \%$ & 2.5 & $9.6 \%$ & $13.1 \%$ & 1.4 & $4.5 \%$ & $8.1 \%$ & 1.9 \\
\hline
\end{tabular}

all p-values $<0.001$

hand are more similar for females (1.8) and males (1.9). Mood and anxiety disorders are more common in females than males in both the reference as well the ADHD group. However, the odds of suffering from mood or anxiety disorders are higher in male ADHD patients than female patients (mood disorders: female 5.0, male 6.4; anxiety disorders: female 3.7, male 4.4). Also, an age specific pattern can be detected: in the age group of over 31 years, the OR for mood and anxiety disorder is nearly twice as high for males compared to females. Also, the occurrence of all four conditions increases with age for individuals from the reference group as well as for ADHD patients. Despite an increase in comorbidities with age in both groups, mood and anxiety disorders in the age group 31 + years still occur over 4 to 9 times more frequently in ADHD patients than in reference patients and anxiety disorders are 2 to 4 times more frequent in ADHD patients than reference patients. This indicates that $65 \%$ of the male and $70 \%$ of the female ADHD patients aged over 31 years in our sample suffer from a mood or anxiety disorder. SUD is 3 to 4 times more prevalent in ADHD patients compared to individuals from the reference group in the age group $31+$ years.

As indicated in Table 4, the noticeably higher occurrence of SUD, obesity, anxiety and mood disorders leads to higher surplus comorbidity-related costs in ADHD patients compared to individuals without ADHD from the reference group but with the same condition. When calculating the cost difference of these groups, ADHD patients show $€ 1420-€ 2715$ higher costs, depending on the comorbid condition. SUD is the most expensive comorbid condition, followed by mood disorders, anxiety disorders, and obesity. The calculated difference in costs between females with ADHD and females without ADHD in comparison to the cost difference between males with ADHD and males without ADHD are noteworthy: The highest surplus costs are detectible for female patients with ADHD for SUD (female: $€ 2821$; male: $€ 2610$ ) and obesity (female: $€ 2038$; male: $€ 1621$ ). Conversely, surplus costs for mood and anxiety disorders are higher in male ADHD patients than female ADHD patients (mood disorders: females $€ 963$, males $€ 1876$; anxiety disorders: females $€ 1535$, males $€ 1792$ ). Stratified by age, none of the four defined comorbidities show the highest costs in the transition age group 18-30 years. Female ADHD patients with SUD or mood disorder show the highest surplus costs in the age group 13-17 years, while obesity and anxiety disorders surplus costs are highest in the age group $\geq 31+$ years. Male ADHD patients have the highest surplus costs in the oldest age group of 31 + years when being diagnosed with SUD. For co-occurring obesity,

Table 4

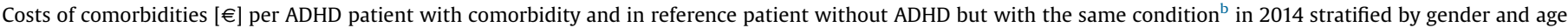
groups.

\begin{tabular}{|c|c|c|c|c|c|c|c|c|c|c|c|c|c|c|c|}
\hline \multirow[b]{2}{*}{ Costs $[€]$} & \multicolumn{3}{|l|}{$0-12$ years } & \multicolumn{3}{|c|}{ 13-17 years } & \multicolumn{3}{|c|}{$18-30$ years } & \multicolumn{3}{|l|}{$\geq 31$ years } & \multicolumn{3}{|c|}{ All age groups } \\
\hline & no ADHD & ADHD & surplus & no ADHD & ADHD & surplus & no ADHD & ADHD & surplus & no ADHD & ADHD & surplus & no ADHD & ADHD & surplus \\
\hline \multicolumn{16}{|l|}{ Female $^{\mathrm{a}}$} \\
\hline SUD & 0 & 0 & 0 & 1,306 & 4,557 & 3,252 & 2,266 & 4,415 & 2,149 & 2,158 & 5,345 & 3,187 & 2,054 & 4,875 & 2,821 \\
\hline Mood disorder & 1,276 & 2,700 & 1,424 & 5,413 & 7,330 & 1,917 & 3,301 & 3,783 & 483 & 3,169 & 4,186 & 1,017 & 3,538 & 4,501 & 963 \\
\hline Anxiety disorder & 996 & 2,322 & 1,326 & 2,182 & 4,216 & 2.033 & 2,029 & 2,789 & 760 & 2,032 & 4,203 & 2,171 & 1,866 & 3,401 & 1,535 \\
\hline Obesity & 596 & 2,001 & 1,405 & 1,101 & 3,067 & 1.965 & 819 & 2,722 & 1,903 & 2,254 & 5,881 & 3,628 & 1,242 & 3,280 & 2,038 \\
\hline \multicolumn{16}{|l|}{ Male $^{a}$} \\
\hline SUD & 722 & 3,528 & 2,807 & 2,153 & 5,138 & 2.984 & 2,463 & 5,076 & 2,613 & 2,146 & 5,148 & 3,003 & 2,440 & 5,050 & 2,610 \\
\hline Mood disorder & 1,574 & 5,131 & 3,557 & 3,601 & 5,150 & 1.549 & 2,412 & 4,103 & 1,692 & 2,518 & 4,124 & 1,607 & 2,603 & 4,479 & 1,876 \\
\hline Anxiety disorder & 1,406 & 3,526 & 2,120 & 2,113 & 3,366 & 1.254 & 926 & 2,903 & 1,977 & 2,544 & 4,034 & 1,491 & 1,649 & 3,441 & 1,792 \\
\hline Obesity & 735 & 2,911 & 2,175 & 832 & 2,187 & 1.355 & 2,439 & 2,610 & 172 & 2,865 & 4,645 & 1,780 & 1,132 & 2,753 & 1,621 \\
\hline \multicolumn{16}{|l|}{ Both gender } \\
\hline SUD & 361 & 1,764 & 1,403 & 1,729 & 4,848 & 3.118 & 2,365 & 4,746 & 2,381 & 2,152 & 5,246 & 3,095 & 2,247 & 4,963 & 2,715 \\
\hline Mood disorder & 1,425 & 3,916 & 2,491 & 4,507 & 6,240 & 1.733 & 2,856 & 3,943 & 1,087 & 2,843 & 4,155 & 1,312 & 3,070 & 4,490 & 1,420 \\
\hline Anxiety disorder & 1,201 & 2,924 & 1,723 & 2,147 & 3,791 & 1.644 & 1,477 & 2,846 & 1,368 & 2,288 & 4,118 & 1,831 & 1,758 & 3,421 & 1,663 \\
\hline Obesity & 666 & 2,456 & 1,790 & 966 & 2,627 & 1.660 & 1,629 & 2,666 & 1,038 & 2,559 & 5,263 & 2,704 & 1,187 & 3,016 & 1,830 \\
\hline
\end{tabular}

a All p-values $<0.001$.

b ADHD and reference group without ADHD 1:1 age and gender adjusted. 
mood and anxiety disorders, surplus costs are highest the in the age group of $0-12$ years.

\section{Discussion}

\subsection{Key findings}

The aim of this study was to estimate the medical costs of ADHD, especially with a focus on the full lifespan and with respect to costs for comorbid conditions (mood, anxiety and substance use disorders, obesity) and stratified by gender. The surplus costs of care for ADHD patients (in comparison to costs of care for persons without ADHD) amount to $€ 1549$ per female and $€ 1467$ per male ADHD patient annually (averaged across every age group). Furthermore, ADHD patients suffer significantly more often from mood and anxiety disorders, SUD, and obesity as compared to the reference group, which is especially visible in adult ADHD patients. Hence, the cost difference between patients with ADHD and a comorbidity and patients without ADHD but the same condition is high, amounting from $€ 1420$ (mood disorder), €1663 (anxiety disorder), $€ 1830$ (obesity) up to $€ 2715$ (SUD).

\subsection{Discussion of findings}

The average costs triggered by health claims of ADHD patients exceed the costs of claims by individuals without ADHD substantially. However, considering age-specific costs in ADHD patients, children incur less surplus costs than adults, which is in accordance with other German and international studies $[3,17,18]$. In our very detailed cost analysis it is noteworthy though that the main costs driver for ADHD patients is not the stimulant treatment of ADHD. Stimulants, which are recommended as medical treatment according to guidelines, account only for a very small proportion of the overall surplus costs of ADHD. This is likely caused by first, moderate prices for stimulants, and second, by the low rate of ADHD medication in adults, as recognized previously [8]. Also, there seems to be a gap of care in the transition age group 18-30 years. This should be seen in light of a strong drop in administrative prevalence in this age group and beyond, and further that in older patient groups, ADHD was not diagnosed during childhood as the diagnosis did not exist. These topics are discussed in more detail below, as stimulant costs decrease notably compared to the age group of teenagers and again increase in adults older than 31 years.

The occurrence of SUD, mood, anxiety disorder, and obesity are higher in the ADHD group compared to the reference group. Again, these findings are consistent with other German and international studies $[3,15,17,20]$. Hence, the novelty of our study lies in the detailed subgroup analyses of comorbidities and the surplus costs for these comorbidities: the costs of the four pre-defined comorbidities of ADHD are significantly higher in patients with ADHD than in individuals suffering from these conditions but without ADHD. These analyses further show that the excess costs in older patients with ADHD compared to younger patients with ADHD are due to ADHD with comorbid conditions. As the costs of comorbid conditions of ADHD, especially for adult ADHD, have not yet been investigated elsewhere, we recommend more research on this topic in other European countries.

In our subgroup analyses we also find several gender differences in the co-occurrence patterns of anxiety and mood disorders, SUD, and obesity: female ADHD patients have a higher prevalence of co-morbid SUD than male ADHD patients, whereas in the reference group, male patients are more often diagnosed with SUD than females. Conversely, male ADHD patients have a higher OR for mood and anxiety disorders than female ADHD patients, whereas mood disorders and anxiety disorders are more common in female individuals without ADHD than males. ADHD thus seems to go against the well-described gender differences in mental disorders (mood and anxiety disorders are more frequent in females, while SUD is more frequent in males); overall prevalence rates are much more similar in ADHD patients than in the reference population. Therefore, ADHD may be a gender-independent risk factor for these comorbidities; the reasons for this however are unclear. Moreover, the rapid increase of ORs in the age group $\geq 31$ years should be studied further in the interest of, firstly, detecting entry points to introduce preventive measures against ADHD associated comorbidities in adults and secondly, to reduce comorbiditydriven healthcare costs. When considering cost-influencing factors beyond direct costs, criminality and loss of productivity are to be considered as well. Further, it is important to keep in mind that indirect costs add to the financial burden to both the patients themselves and to society [19]. Overall, more research in the general population using diagnostic instruments rather than only the patient's referral status to estimate gender and age differences in the comorbid conditions of ADHD over the lifespan is needed to complement our findings.

Besides the high level of costs for ADHD patients, which is substantial, it is interesting that we see a drop of costs between the ages of 18 and 30 years and a strong increase thereafter. This suggests a gap of care for ADHD patients in the transition from childhood to adulthood, which has been dubbed the 'transition gap' [21]. This finding is consistent with findings from other German claims data studies $[9,17]$. Reasons for the transition gap can be manifold: omission of regular check-ups by a pediatrician after ADHD patients come of age, poor cooperation between adolescent and adult psychiatry, moving out from home, which tends to lead to less health literacy and intermittingly decreased awareness of ADHD core symptoms e.g. during the first years of university and/ or vocational training, to name but a few. However, beyond early adulthood the costs per person rise, often because of comorbid conditions. It is intriguing to speculate that these comorbidities, which were shown to be important cost drivers in adulthood, could be prevented if mental healthcare were provided more constantly over the lifespan. Preventing the development of comorbidities with age should be the focus of mental health care. Early treatment starting in childhood and continued treatment of adolescents into adulthood seem therefore advisable. There is first evidence of protective effects of stimulant treatment in individuals with ADHD with regard to unintentional injuries, suicidal behavior, SUDs, and depression [22-25]. This calls for a better planned and better executed transition period, offering both stimulant treatment and nondrug-based services to ADHD patients, as proposed previously [28]. Overall, services for (young) adult ADHD patients must be made available beyond sectoral barriers. Further, awareness of the individual needs of (young) adult ADHD patients, which may differ between men and women and in different age groups, needs to rise and more individualised care programmes are recommendable [26]. Training relevant professionals, including primary care physicians, in recognizing adult ADHD and individual needs with a special regard to the transition age group is an important step. By introducing primary care physicians it would be possible to close the gap in care for ADHD patients, regardless of age [27]. Many patients may profit from such individualised care programmes, which have also shown in the past to have cost-cutting effects [29]. As it will be more and more important for SHIs to allocate resources thoughtfully, i.e. preventing exploding costs while at the same time improving the quality of care, individualised care programmes will gain importance and will benefit from detailed knowledge on cost drivers. 


\subsection{Strengths and limitations}

A major strength of this study is the large sample size, with approximately 4 million member-records available from over 70 German SHIs. This makes robust analyses with a very large ADHD cohort and reference group possible. Moreover, these results can be extrapolated to other individuals diagnosed with ADHD in Germany, as the sample derived from the database is highly representative. Healthcare claims, medical costs and comorbidities are stratified by age and gender. Therefore, the study gives a rich insight into costs of adult ADHD patients and the comorbid conditions that have so far rarely been documented with real world data.

A first limitation of our study is that only four comorbid conditions, which were defined a priori and chosen because of their high prevalence in ADHD patients but do not depict all cooccurring conditions associated with ADHD, are analysed in detail. Moreover, comorbid obesity is likely underestimated, given that only extreme obese individuals are diagnosed and coded as such. Note, however, that the findings on the overall healthcare costs of ADHD include the direct costs of all comorbidities.

Another, more important, limitation is that we can only assess the administrative ADHD prevalence rate (as opposed to the epidemiological prevalence rate), which likely leads to a systematic underestimation of patients with ADHD: in routine billing data, only documented and diagnosed patients are observed. When comparing our ADHD prevalence rates to epidemiological studies, it becomes obvious that a high number of patients remain unrecorded, especially for adults who were not diagnosed in childhood. The presence of unrecorded patients is illustrated by the overall lifespan prevalence of $0.68 \%, 2.9 \%-4.1 \%$ in childhood and $0.1 \%$ to $0.7 \%$ in adulthood, which is low compared to worldwide estimates of $5.3 \%$ in childhood and $3.4 \%$ in adulthood [7,30]. Also, this biases our study to more severe cases and especially in adulthood, ADHD patients may likely enter the healthcare system because of their comorbid condition and not their ADHD as such, which yields an overestimation of comorbidity and costs in adulthood. However, the fact that adults seek help for other psychiatric conditions implies that ADHD may still be inadequately diagnosed upon referral (e.g. because the comorbid condition cloaks ADHD or dominates the clinical picture), which leads to an underestimation of ADHD costs. On the other hand, adult ADHD patients without comorbid conditions may not enter the mental healthcare sector at all and hence might end up undiagnosed. This however underscores the notion that comorbidity in ADHD may be the relevant cost driver and hence needs close attention.

Overall, given that we relied on claims data on ADHD patients with a diagnosis in the inpatient setting or two secured diagnosis in the outpatient setting in two different quarters of a year, there may be additional biases in our cost estimates. The surplus costs of ADHD adjusted for the general population may be substantially lower, as the diagnosed ADHD patients may be the more severe cases, which more often seek treatment and are more costintensive than less severe forms of ADHD. This indicates that our estimates of the mean (surplus) costs per patient are biased upwards, particularly in adult ADHD. In the age group $\geq 31$ years, ADHD was not diagnosed during childhood as the diagnosis did not exist. Therefore, adult patients with ADHD may often seek help for the comorbid condition rather than ADHD itself, but receive a codiagnosis of hitherto undetected ADHD. Our reasoning would imply that in adulthood comorbid patients are over-represented, and costs are over-estimated. It is important to note that all studies that rely on referred cases have these biases. Future studies with bottom-up measurement of ADHD, comorbid conditions, and healthcare use would be an important addition to the current literature, providing insight into the extent of bias in the current literature. Overall, it has to be kept in mind that claims data are recorded for billing purposes. Their use as a data source is limited as we do not know why individuals made use of different health services and resources.

Further, minor, limitations of our study are, first, that we could not include out-of-pocket payments in our cost estimates, as claims data presents a public sick fund perspective only; this however rarely applies as public sick funds usually pay for all needed medical procedures necessary. Second, patients with the ICD-code F90.9 are excluded due to unspecific coding, which reduces the number of patients by $10 \%$. Only continuously insured persons throughout the observation period, except individuals who died within this period, are included in the analyses, which reduces the patient number by $5 \%$. These decisions in inclusion and exclusion criteria may have enhanced the underestimation of ADHD in the database.

\subsection{Conclusion}

Despite these limitations, we are among the first studies to thoroughly consider the costs incurred in childhood and adult ADHD and the administrative prevalence of comorbid conditions. We conclude that costs are high, with these comorbid conditions of ADHD being important cost-drivers, and even though biased upwards, costs are likely to increase in the coming years. Furthermore, we are able to identify a gap in care, which should lead to a better cooperation between different kinds of care providers ensuring continuous treatment and a better awareness of ADHD symptoms in young adults and adults.

\section{Declaration of interest}

No competing interests are disclaimed.

\section{Funding}

Financial support for this study was received from the European Union's Horizon 2020 research and innovation programme under grant agreement 667302 (Comorbid Conditions of ADHD (CoCA). Prof. Dr. A. Reif received personal fees from MEDICE Arzneimittel Pütter GmbH \& Co. KG, Shire PLC, neuraxpharm Arzneimittel $\mathrm{GmbH}$, Janssen-Cilag $\mathrm{GmbH}$ and Servier Deutschland $\mathrm{GmbH}$ present or during 36 months prior to publication. No competing interest are disclaimed.

\section{References}

[1] Kooij SJJ, Bejerot S, Blackwell A, Caci H, Casas-Brugué M, Carpentier PJ, et al. European consensus statement on diagnosis and treatment of adult ADHD: the European network adult ADHD. BMC Psychiatry 2010;10:67.

[2] Gupte-Singh K, Singh RR, Lawson KA. Economic burden of attention-deficit/ hyperactivity disorder among pediatric patients in the United States. Value Health 2017;20(4):602-9.

[3] Braun S, Zeidler J, Linder R, Engel S, Verheyen F, Greiner W. Treatment costs of attention deficit hyperactivity disorder in Germany. Eur J Health Econ 2016;17:371-3.

[4] Katzman MA, Bilkey TS, Chokka PR, Fallu A, Klassen LJ. Adult ADHD and comorbid disorders: clinical implications of a dimensional approach. BMC Psychiatry 2017; 17:302.

[5] Hodgkins P, Montejano L, Sasané R, Huse D. Cost of illness and comorbidities in adults diagnosed with attention-deficit/hyperactivity disorder: a retrospective analysis. Primary Care Comp CNS Disorders 2011;13(2).

[6] Visser S, Danielson M, Bitsko R, Holbrook J, Kogan M, Ghandour R, et al. Trends in the parent-report of healthcare provider diagnosed and medicated ADHD: United States, 2003-2011. J Am Acad Child Adolesc Psychiatry 2014;53:34-46.

[7] Fayyad J, De Graaf R, Kessler R, Alonso J, Angermeyer M, Demyttenaere K, et al. Cross-national prevalence and correlates of adult attention-deficit hyperactivity disorder. Br J Psychiatry 2007;190:402-9.

[8] Fayyad J, Sampson N, Hwang I, Adamowski T, Aguilar-Gaxiola S, Al-Hamzawi A et al. ADHD attention deficit and hyperactivity disorders - the descriptive epidemiology of DSM-IV adult ADHD in the World Health Organization. Atten Defic Hyperact Disord 2017;9:47-65. 
[9] Bachmann C, Philipsen A, Hoffmann F. ADHS in Deutschland: trends in diagnose und medikamentöser therapie [ADHD in Germany: trends in diagnosis and pharmacotherapy]. Deutsches Ärzteblatt 2017;114:141-8.

[10] Wehmeier P, Schacht A, Rothenberger A. Change in the direct cost of treatment for children and adolescents with hyperkinetic disorder in Germany over a period of four years. Child Adolesc Psychiatry Mental Health 2009;2009(3):3.

[11] Jacob C, Gross-Lesch S, Reichert S, Geissler J, Jans T, Kittel-Schneider S, et al. Sex- and subtype-related differences of personality disorders (axis II) and personality traits in persistent ADHD. J Atten Disord 2014;20:1056-65.

[12] Cook B, Li D, Heinrich K. Obesity, physical activity, and sedentary behavior of youth with learning disabilities and ADHD. J Learn Disabil 2014:48:563-76.

[13] Khalife N, Kantomaa M, Glover V, Tammelin T, Laitinen J, Ebeling H, et al. Childhood attention-deficit/hyperactivity disorder symptoms are risk factors for obesity and physical inactivity in adolescence. J Am Acad Child Adolesc Psychiatry 2014;53:425-36.

[14] Klora M, Zeidler J, Linder R, Verheyen F, von der Schulenburg J. Costs and treatment patterns of incident ADHD patients - a comparative analysis before and after the initial diagnosis. Health Econ Rev 2015;5:40.

[15] Kessler R, Adler L, Berglund P, Green J, McLaughlin K, Fayyad J, et al. The effects of temporally secondary co-morbid mental disorders on the associations of DSM-IV ADHD with adverse outcomes in the US National Comorbidity Survey Replication Adolescent Supplement (NCS-A). Psychol Med 2014;44:1779-92.

[16] Anderson F, Walker J. Characteristics and external validity of the German Health Risk Institute (HRI) database. Pharmacoepidemiol Drug Saf 2015;25:106-9.

[17] Klora M, Zeidler J, Lublow D, Linder R, Verheyen F, von der Schulenburg M. Ageand gender-specific costs as well as drug therapies of ADHD patients. Gesundheitswesen 2015;78:23-9.

[18] Birnbaum H, Kessler R, Lowe S, Secnik K, Greenberg P, Leong S, et al. Costs of attention deficit-hyperactivity disorder (ADHD) in the US: excess costs of persons with ADHD and their family members in 2000. Curr Med Res Opin 2005;21:195-205.

[19] Daley D, Hojberg-Jacobsen R, Lange A, Sorensen A, Walldorf J. Costing adult attention deficit hyperactivity disorder - impact on the individual and society. Oxford University Press; 2015.
[20] Instanes J, Klungsoyr K, Halmoy A, Fasmer O, Haavik J. Adult ADHD in comorbid somatic disease: a systematic literature review. J Attent Disorders 2016;22:203-28.

[21] Singh S, Tuomainen H. Transition from child to adult mental health services: needs, barriers, experiences and new models of care. World Psychiatry $2015 ; 14: 358-61$.

[22] Ruiz-Goikoetxea M, Cortese S, Aznarez-Sanado M, Magallón S, Alvarez Zallo N, Luis EO, et al. Risk of unintentional injuries in children and adolescents with ADHD and the impact of ADHD medications: a systematic review and metaanalysis. Neurosci Biobehav Rev 2018;84:63-71.

[23] Chang Z, Lichtenstein P, Halldner L, D'Onofrio B, Serlachius E, Fazel S, et al. Stimulant ADHD medication and risk for substance abuse. J Child Psychol Psychiatry 2014;55(8):878-85.

[24] Chen Q, Sjölander A, Runeson B, D’Onofrio BM, Lichtenstein P, Larsson H. Drug treatment for attention-deficit/hyperactivity disorder and suicidal behaviour: register based study. BMJ 2014;18(348):g3769.

[25] Chang Z, D’Onofrio BM, Quinn PD, Lichtenstein P, Larsson H. Medication for attention-deficit/hyperactivity disorder and risk for depression: a nationwide longitudinal cohort study. Biol Psychiatry 2016;15(12)916-22 80.

[26] Taylor N, Fauset A, Harpin V. Young adults with ADHD: an analysis of their service needs on transfer to adult services. Arch Dis Child 2010;95:513-7.

[27] Fegert JM, Hauth I, Banaschewski T, Freyberger HJ. Übergang zwischen Jugend und Erwachsenenalter: Herausforderungen für die Transitionspsychiatrie. Zeitschrift für Kinder- und Jugendpsychiatrie und Psychotherapie 2017;45 (1):80-5.

[28] Young S, Murphy Clodagh M, Coghill D. Avoiding the 'twilight zone' recommendations for the transition of services from adolescence to adulthood for young people with ADHD. BMC Psychiatry 2011;11:174

[29] Rabbata S. Integrierte Versorgung: Kooperation im Kinzigtal spart Kosten [Integrated Care: Cost Savings through Cooperation in Kinzigtal]. Deutsches Ärzteblatt 2009;106:A966-8.

[30] Polanczyk G, Silva de Lima M, Lessa-Horta B, Biederman J, Rohde L. The worldwide prevalence of ADHD: a systematic review and metaregression analysis. Am J Psychiatry 2007;164:942-8. 